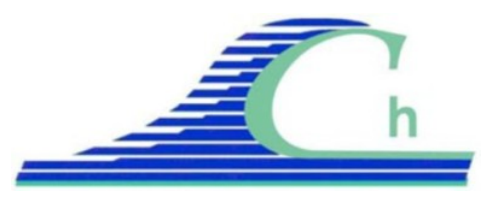

XII ${ }^{\text {èmes }}$ Journées Nationales Génie Côtier - Génie Civil

Cherbourg, 12-14 juin 2012

DOI:10.5150/jngcgc.2012.075-E C Editions Paralia CFL

disponible en ligne - http://www.paralia.fr - available online

\title{
Détermination expérimentale du coefficient de diffusion des ions chlorures dans le béton armé
}

\section{Nora EL AMRI ${ }^{1}$, Mohamed SABBAR ${ }^{2}$, Hamid CHAKIR ${ }^{2}$, Ghita MANGOUB ${ }^{1}$}

1. Faculté des Sciences Techniques de Settat Maroc.

2. Faculté des Sciences Ben M’Sik Casablanca, Maroc.

noraelamri2@gmail.com

\section{Résumé :}

La durabilité des ouvrages en béton armé en milieu marin est liée à la pénétration d'agents agressifs tels que les ions chlorure qui contribuent à la corrosion des armatures. L'évaluation de ce paramètre peut notamment aider au choix de formules de béton adaptées à l'environnement considéré ou contribuer au diagnostic d'ouvrages existants. Dans le présent travail, nous allons décrire une procédure de détermination de ce coefficient applicable au divers béton durcis ayant acquit une résistance notable. Cette procédure est faite au laboratoire par des essais accélérés. La diffusion des chlorures est simulée et les fronts de diffusion ont été calculés.

Mots-clés :

Bétons durcis - Essais destructifs - Essais accélérés - Coefficient de diffusion Perméabilité -Porosité - Durabilité - Ions de chlorures

\section{Introduction}

La corrosion des armatures dans les ouvrages en béton armé est due principalement à la pénétration d'agents agressifs provenant du milieu extérieur à travers la porosité ou les fissures (NGUYEN et al., 2005). Parmi ces agents, figurent principalement les ions chlorures présents dans l'eau de mer. La pénétration est le résultat de plusieurs processus qui diffèrent selon l'état de saturation des matériaux. Dans le cas des ouvrages immergés dans l'eau de mer, la diffusion est le phénomène principal de transport puisque le matériau est en état de saturation totale. Cette diffusion est due à la différence de concentration ionique entre l'extérieur et l'intérieur (DEBY, 2008).

Nous allons présenter plusieurs essais au laboratoire sur les indicateurs de la durabilité du béton durci. Les premiers essais concernent la perméabilité à l’oxygène, les seconds essais concernent la détermination de la porosité et les troisièmes essais sont relatifs à la détermination du coefficient de diffusion qui sera utilisé pour les simulations de l'équation de diffusion.

Le choix des protocoles expérimentaux sont effectués en se référant aux travaux de (CARLIER et al., 2007 ; FOKWA \& BERTHAUD, 2007 ; PERALTA \& BANGI, 2003). 


\section{2- Détermination expérimentale des indicateurs de durabilité}

Pour ces essais, le choix des indicateurs de la perméabilité, de la porosité et la détermination du coefficient de diffusion est fait dans le but de caractériser la durabilité d'un béton durci. Les échantillons sont prélevés par carottage sur plusieurs structures destinées à des ouvrages maritimes. Leurs dimensions sont de $15 \mathrm{~cm}$ de diamètre et de 5 cm d'épaisseur. Ils sont réalisés par découpage sur ouvrage ou sur élément préfabriqué. Sur le tableau 1, les différents types d'ouvrages testés, dans un environnement marin, sont présentés.

Tableau 1. Ouvrages analysés.

\begin{tabular}{ll}
\hline Nature de l'ouvrage & $N^{\circ}$ de référence \\
\hline Pré poutre & $1,3,6$ \\
Palme & $2,4,10,11,14$ \\
poteau & $5,7,9,12,13,15,16$ \\
\hline
\end{tabular}

2.1 Détermination de la perméabilité à l’oxygène

La présente expérience a pour objet de définir l'essai de perméabilité à l'oxygène des corps d'épreuve en béton durci. L'essai consiste à soumettre un corps d'épreuve cylindrique à un gradient de pression constant. La perméabilité est déterminée à partir de la mesure du flux en régime permanent.

Pour la détermination de la perméabilité au gaz spécifique d'un béton donné, les éprouvettes sont recouvertes sur leurs faces latérales d'un film en papier aluminium adhésif et sont étuvés à $105{ }^{\circ} \mathrm{C}$ jusqu'à masse constante. L'appareillage utilisé est un perméamètre à charge constante composé d'une cellule permettant de mesurer la perméabilité de corps d'épreuve de diamètre $15 \mathrm{~cm}$, d'un dispositif comprenant une bouteille d'oxygène et une bouteille d'air industriel munies de détendeurs pouvant maintenir le niveau de pression et de trois débitmètres à bulle.

La formule suivante donne la perméabilité de chaque corps d'épreuve :

$k=\frac{2 \times P_{a} \times Q \times L \times \mu}{A \times\left(P_{0}^{2}-P_{a}^{2}\right)}$

Avec :

$Q=\frac{V}{t_{\text {moy }}}$

$L$ : épaisseur du corps d'épreuve

$k$ : perméabilité

$P_{0}:$ Pression absolue à l'entrée

$P_{a}$ : Pression atmosphérique

$Q$ : débit volumique

$A$ : section du corps d'épreuve 
$V:$ portion volumique entre deux repères du débitmètre à bulle $t_{\text {moy }}:$ moyenne des temps de parcours

$\mu$ : Viscosité dynamique de l'oxygène $\left(2,02 \times 10^{-5} \mathrm{~Pa}\right.$ s à $\left.20^{\circ} \mathrm{C}\right)$

Toutes les variables sont en unités S.I.

Sur la figure 1 les résultats présentés sont relatifs au béton 65, pris par carottage dans les ouvrages du tableau 1 . Le choix des essais relatifs à 7 jours, 28 jours et 56 jours est fait dans un souci de comparaison puisque les résultats de la perméabilité à l'oxygène dépendent du nombre de jours. Il existe un état où la perméabilité à l'oxygène est constante qui est estimé dans ces expériences à 56 jours. En effet, quelques essais ont été effectués auparavant sur quelques carottes à 90 jours et donnent les mêmes tendances.

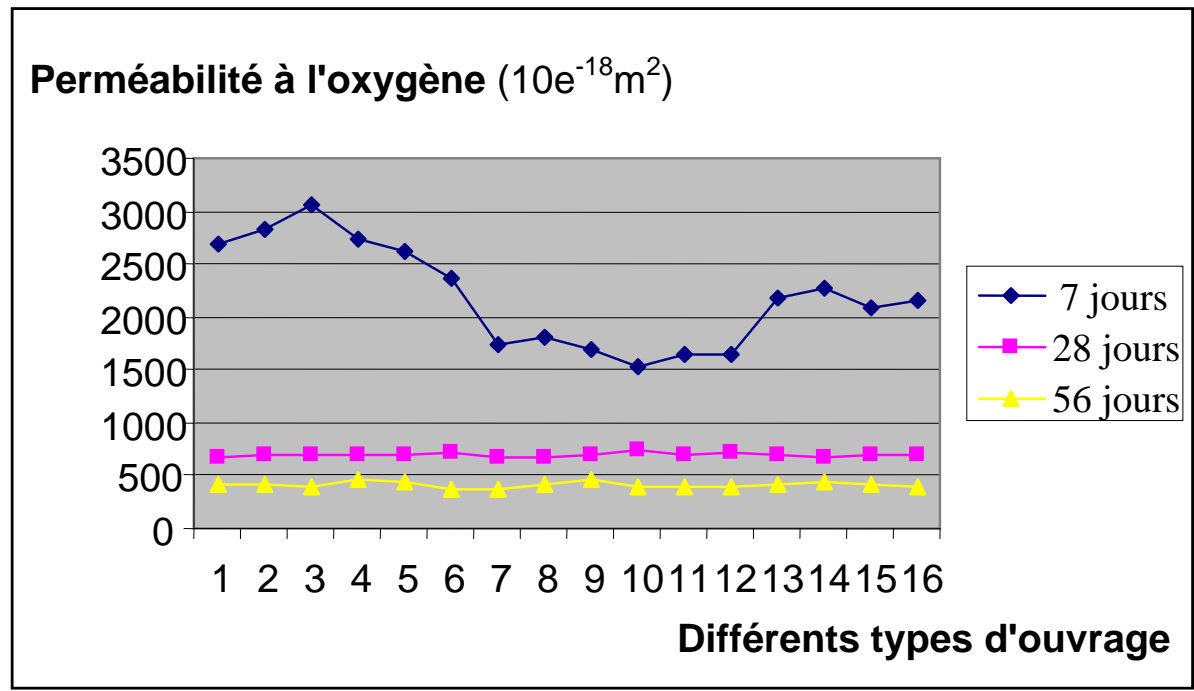

Figure 1. Perméabilité à l'Oxygène pour différents ouvrage et pour un béton 65 .

\subsection{Détermination de la masse volumique et de la porosité accessible à l'eau}

L'objet de l'essai est la détermination de la masse volumique apparente, la porosité ouverte et la porosité totale. La porosité totale est la somme de la porosité ouverte et la porosité fermée. La porosité ouverte est la porosité accessible à l'eau. Elle représente le rapport du volume total des pores ouverts dans un corps d'épreuve à son volume apparent. La porosité fermée est le rapport du volume total des pores qui ne sont pas pénétrés par le liquide d'immersion à son volume apparent.

L'appareillage est constitué d'une étuve, d'une balance hydrostatique, d'un appareillage à vide, d'un thermomètre, d'un liquide d'imbibition, d'un dessiccateur et d'un récipient de volume suffisant pour permettre l'immersion totale du corps d'épreuve.

Les expressions utilisées sont :

$E=\frac{M_{\text {air }}-M_{\text {sec }}}{M_{\text {air }}-M_{\text {eau }}} * 100$ 
$\rho_{d}=\frac{M_{\mathrm{sec}}}{M_{\text {air }}-M_{\text {eau }}}$

$\rho_{d} \quad$ La masse volumique apparente.

E La porosité accessible à l'eau.

$M_{\text {eau }} \quad$ La masse du corps d'épreuve immergé dans l'eau.

$M_{\text {air }} \quad$ La masse, du corps d'épreuve imbibé.

$M_{\text {sec }} \quad$ La masse du corps d'épreuve après étuvage à $105^{\circ} \mathrm{C}$.

Pour les mêmes ouvrages mentionnés dans le tableau 1 , la porosité est présentée sur la figure 2. Ces résultats permettent de constater que la porosité reste du même ordre de grandeur pendant la durée de 56 jours.

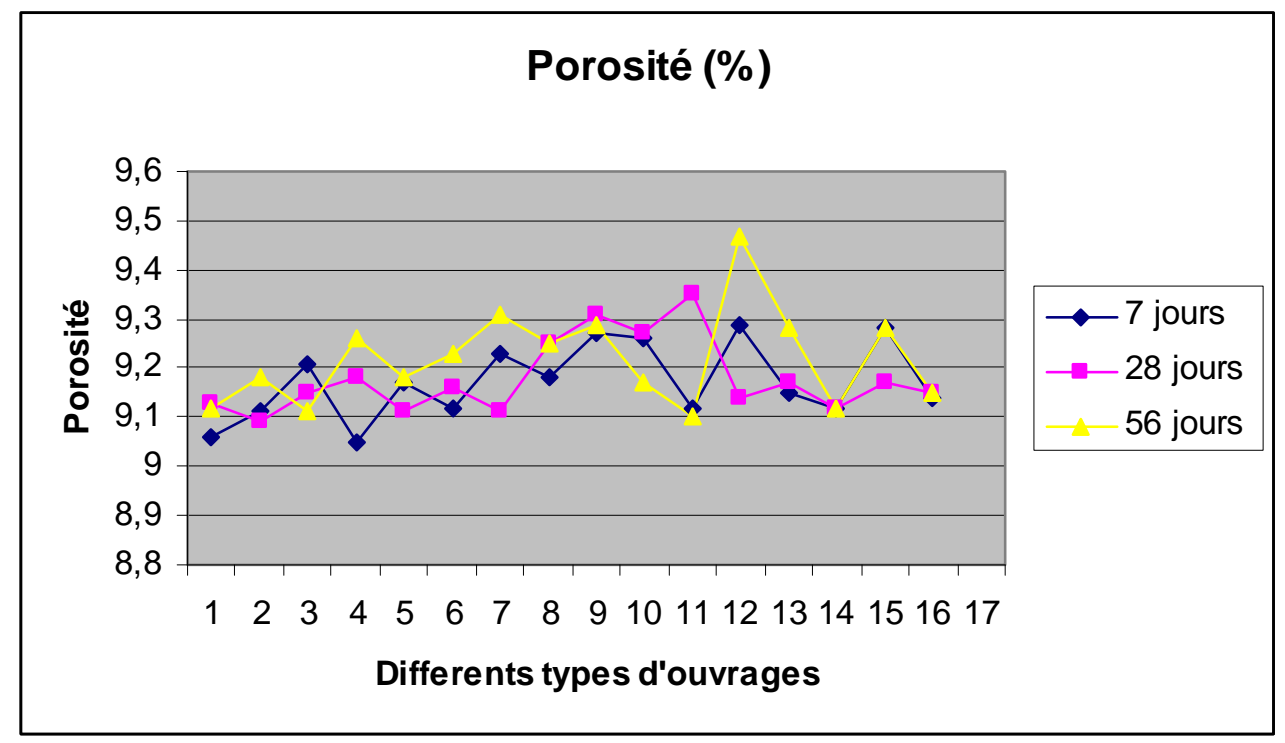

Figure 2. La porosité pour différents ouvrages en béton 65.

\subsection{Détermination du coefficient de diffusion des chlorures dans le béton durci}

Dans cet essai, le coefficient de diffusion des ions chlorures dans le béton durci est déterminé. Ce béton est un béton ayant acquis une résistance notable

Trois éprouvettes de dimensions $15 \mathrm{~cm}$ et $5 \mathrm{~cm}$ sont réalisées et découpées d'une manière humide. Après essuyage avec un chiffon, les éprouvettes sont soumises sous vide pendant 3 heures. Ensuite les éprouvettes sont immergées dans une solution saturée de $\mathrm{Ca}(\mathrm{OH})_{2}$ pendant 18 heures. On présente sur la figure 3 l'appareillage utilisé pour les essais.

La mesure de la profondeur de pénétration des chlorures se fait de la manière suivante : Un potentiel électrique externe est appliqué à travers l'échantillon et force les ions chlorures à migrer dans l'échantillon. L'éprouvette est découpée en deux et ce parallèlement à son axe. La solution de nitrate d'argent $0,1 \mathrm{M}$ est étalée sur une des sections obtenues. Après environ 15 minutes, la profondeur de pénétration des chlorures 


\section{XII ${ }^{\text {èmes }}$ Journées Nationales Génie Côtier - Génie Civil \\ Cherbourg, 12-14 juin 2012}

sera visible. De part et d'autre du centre de la section testée, la mesure, de la profondeur de pénétration, est faite en 7 points espacés de $10 \mathrm{~mm}$.

Les résultats des essais relatifs à la diffusion des ions de chlorure dans le béton durci sont présentés sur la figure 4 .
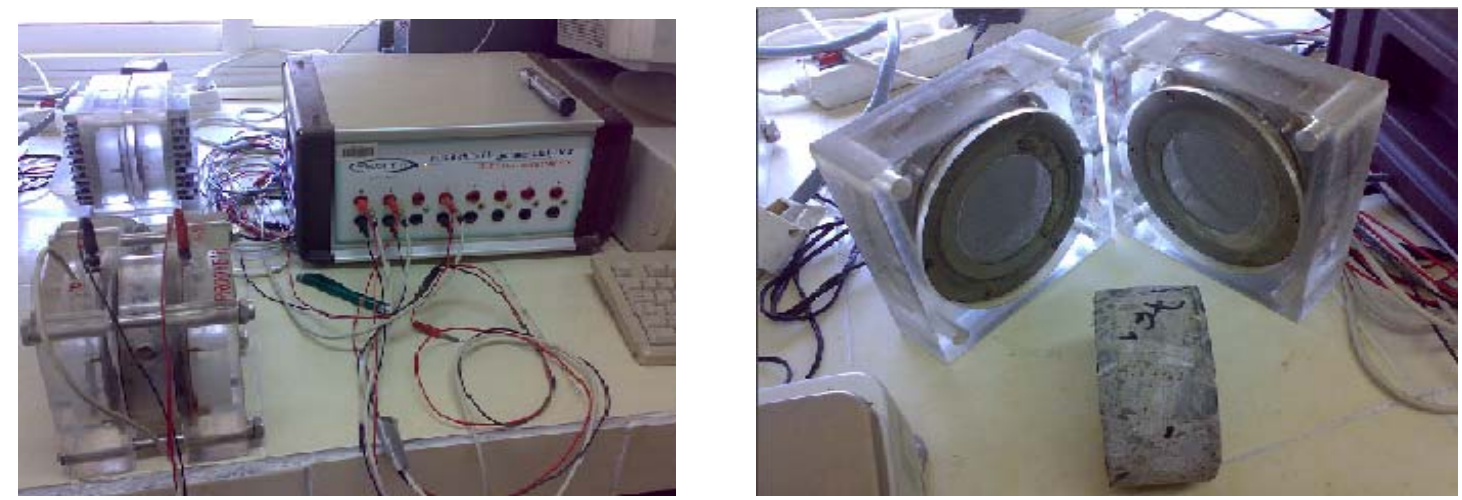

Figure 3. Dispositif expérimental de la diffusion des chlorures.

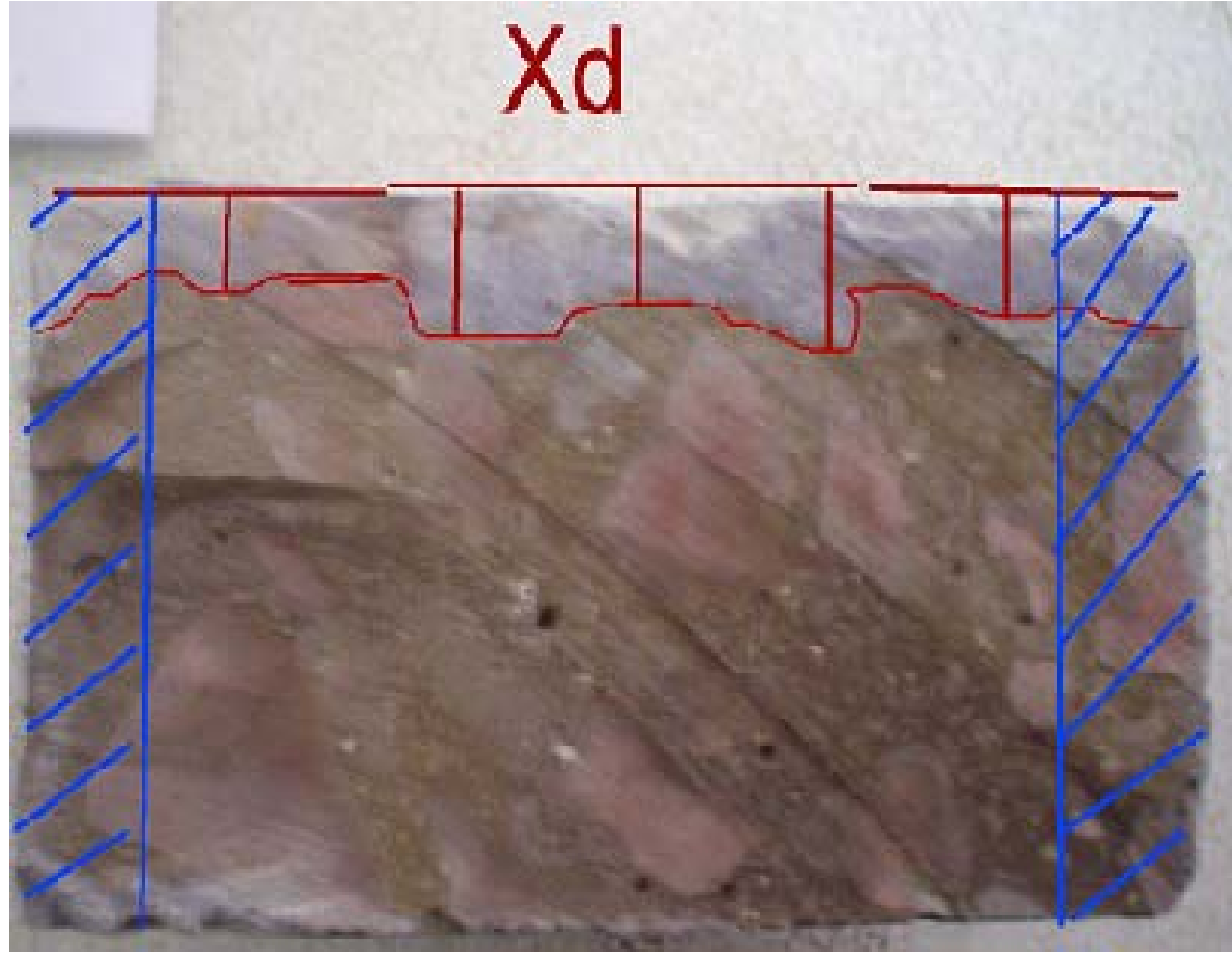

Figure 4. Front de la diffusion.

Le coefficient de diffusion est calculé à partir de l'équation :

$D_{\text {nssm }}=\frac{0,0239 \times(273+T) \times L}{(U-2) \times t}\left(x_{d}-0,0238 \times \sqrt{\frac{(273+T) \times L \times x_{d}}{U-2}}\right)$

Avec : 
$D_{n s s m}$ : Coefficient de diffusion en régime non stationnaire en $\mathrm{m}^{2} / \mathrm{s}$

$T: \quad$ Température moyenne des températures initiale et finales en ${ }^{\circ} \mathrm{C}$

$L: \quad$ Epaisseur de l'échantillon en $\mathrm{mm}$

$U: \quad$ Tension appliquée en $\mathrm{V}$

$x_{d}: \quad$ Valeur moyenne de la pénétration des chlorures en mm

$t$ : $\quad$ Durée du test en heures

Les résultats du coefficient de diffusion sont présentés sur la figure 5 . La valeur utilisée pour la simulation numérique est $2,5 \times 10^{-12} \mathrm{~m}^{2} / \mathrm{s}$.



Figure 5. Résultats du coefficient de diffusion.

\section{Equation de diffusion}

Le coefficient de diffusion $D$ est la constante de proportionnalité entre le flux $J$ d'une particule et le gradient de sa concentration $C$. De ce postulat, Fick en écrivit sa première équation :

$\vec{J}=-D \overrightarrow{\text { grad }} C$

Cette loi a été énoncée par Fick aussi sous la forme de l'équation unidimensionnelle suivante :

$$
\frac{\partial}{\partial t} C(x, t)=D \frac{\partial^{2}}{\partial x^{2}} C(x, t)
$$

Dans le cas bidimensionnel, l'équation de diffusion s'écrie en coordonnées cartésiennes sous la forme:

$$
\frac{\partial}{\partial t} C(x, y, t)=D\left(\frac{\partial^{2}}{\partial x^{2}} C(x, y, t)+\frac{\partial^{2}}{\partial y^{2}} C(x, y, t)\right)
$$

La solution est donnée par :

$$
C(x, y, t)=\frac{A}{4 \pi D t} \exp \left[\frac{-\left(x^{2}+y^{2}\right)}{4 D t}\right]
$$




\section{XII ${ }^{\text {èmes }}$ Journées Nationales Génie Côtier - Génie Civil \\ Cherbourg, 12-14 juin 2012}

La représentation qualitative faite sur la figure 6 montre l'évolution du phénomène de diffusion à titre indicatif. En effet, pour une valeur initiale de la concentration introduite au centre de la section ( $C=1$ par exemple), la diffusion se produit d'une manière symetrique de part et d'autre du centre.

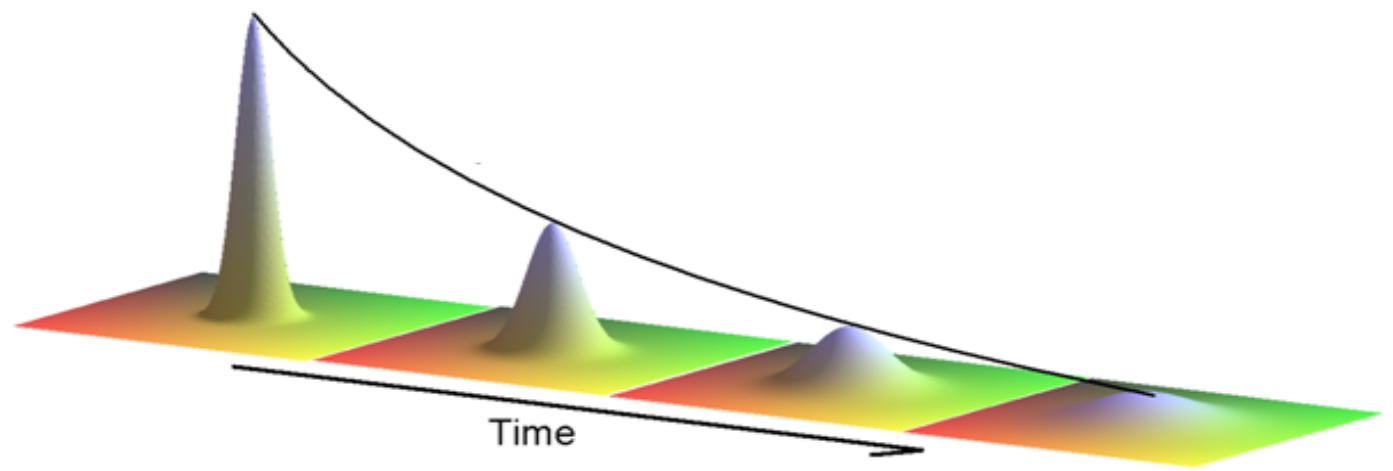

Figure 6. Schématisation de la diffusion bidimensionnelle

Les particules localisées à l'endroit où elles étaient introduites diffusent vers les voisinages de leurs emplacements d'origine de façon à homogénéiser la répartition.

\section{Conclusion et perspectives}

Les indicateurs de durabilité et en particulier le coefficient de diffusion sont aujourd'hui des paramètres indispensables dans l'évaluation des durées de vie des ouvrages en béton armé. Ils jouent un rôle majeur dans la modélisation des structures et dans la détermination de l'épaisseur d'enrobage qui répand aux exigences demandées.

Reste à noter que les modes opératoires ainsi que les solutions analytiques ne sont désormais pas standards. La résolution analytique représente une vaste gamme de méthodologies allant des plus simples aux plus compliquées mais qui sont toutes basées sur des approximations. Les expériences que nous avons représentées sont basées sur la norme européenne, elles utilisent des approximations allant dans le sens de la sécurité et donnent des résultats fiables servant d'élément de base pour la modélisation des ouvrages en béton.

Une modélisation numérique sera indispensable pour prévoir le comportement du milieu. On aura aussi à réfléchir sur les modes expérimentaux existants pour la détermination du coefficient de la diffusion et voir les possibilités de mise en évidence d'autres modes non destructifs et réalisables. 
Thème 4-Ouvrages portuaires et offshore

\section{Références}

CARLIER J.-P., FAN-CHAO G., BURLION N. (2007). Evaluation expérimentale des propriétés hydrodynamiques des matériaux cimentaires. $18^{\text {ème }}$ Congrès français de mécanique, Grenoble, 27-31 août.

DEBY M.F. (2008). Approche probabiliste de la durabilité des bétons en environnement marin. Thèse de doctorat de l'université de Toulouse.

FOKWA D., BERTHAUD Y. (2007). Mesure de la ténacité du béton par corrosion accélérée et corrélation d'images. $18^{\text {ème }}$ Congrès français de mécanique, Grenoble, 2731 août.

NGUYEN Q.T., CARE S., BERTHAUD Y., MILLARD A. (2005). Fissuration $d u$ béton soumis à la corrosion. L'objet. Volume 8, $\mathrm{n}^{\circ}$ 2, pp 1-15.

NGUYEN Q.T., BERTHAUD Y., CARE S., L'HOSTIS V., MILLARD A. (2005). Etude expérimentale et modélisation du couplage corrosion - fissuration dans le béton armé. $17^{\text {ème }}$ Congrès français de mécanique, Troyes, 29 août -2 septembre.

PERALTA P.N., BANGI A.P. (2003). A nonlinear regression technique for calculating the average diffusion coefficient of wood during drying. Wood and Fiber Science, (3), pp 401- 408. 